\title{
Valores da exoftalmometria média na população adulta da região do Grande ABC, São Paulo/Brasil
}

\section{Normal exophthalmometric values in adult population of ABC Region, Sao Paulo/Brazil}

Nadia Ajub Moysés ${ }^{1}$, Lúcia Míriam Dumont Lucci² ${ }^{2}$ Nilson Lopes da Fonseca Júnior , José Ricardo Carvalho Lima Rehder ${ }^{4}$

\section{ResUmo}

Objetivo: Estabelecer padrões de normalidade dos valores da exoftalmometria de ambos os olhos e da distância entre os rebordos orbitários laterais (base) em adultos jovens da população do Grande ABC São Paulo/Brasil. Métodos: Realizou-se a exoftalmometria com o exoftalmômetro de Hertel, em 129 pacientes do sexo feminino e 75 do sexo masculino, na faixa etária de 25 a 55 anos. Os pacientes foram separados em três grupos raciais (brancos, negros e pardos). Resultados: A variação da exoftalmometria encontrada na população em estudo foi de 10 a 23mm, média de 15,57 $\pm 2,43 \mathrm{~mm}$. No sexo feminino a média foi de $15,42 \pm 2,40 \mathrm{~mm}$ e no masculino, 15,83 $\pm 2,47 \mathrm{~mm}$. Não se encontrou uma relação estatisticamente significativa entre os sexos feminino e masculino $(p=0,25)$. Na raça branca a média foi de $15,57 \pm 2,46 \mathrm{~mm}$, na negra foi de $16,20 \pm 2,70 \mathrm{~mm}$ e na parda foi de $15,02 \pm 2,01 \mathrm{~mm}$. A diferença foi estatisticamente significativa $(\mathrm{p}=0,008)$ entre as raças negra e parda. A medida da base variou de 104 a $125 \mathrm{~mm}$ (média de $113,91 \pm 4,37 \mathrm{~mm}$ ). A diferença entre homens e mulheres foi estatisticamente significativa $(\mathrm{p}<0,001)$. Na raça branca a média da base encontrada foi de $112,18 \pm 4,02 \mathrm{~mm}$, na negra foi de $115,37 \pm 4,71 \mathrm{~mm}$ e na parda, $115,18 \pm 3,66 \mathrm{~mm}$. A diferença da média da base foi estatisticamente significativa $(\mathrm{p}<0,001)$ entre as raças branca e negra e branca e parda. Nenhum paciente apresentou assimetria entre os dois olhos maior que $2 \mathrm{~mm}$. Conclusão: $O$ padrão de normalidade da exoftalmometria encontrado para a população em estudo foi de 10 a 23mm e a base variou de 104 a $125 \mathrm{~mm}$.

Descritores:Exoftalmia/diagnóstico; Técnicas de diagnóstico oftalmológico/ instrumentação; Valores de referência

\footnotetext{
${ }^{1}$ Estagiária do Setor de Cirurgia Plástica Ocular da Disciplina de Oftalmologia da Faculdade de Medicina do ABC - FMABC - Santo André (SP) - Brasil;

${ }^{2}$ Doutora, Médica Colaboradora do Setor de Cirurgia Plástica Ocular da Disciplina de Oftalmologia da Faculdade de Medicina do $\mathrm{ABC}$ - FMABC - Santo André (SP) - Brasil;

${ }^{3}$ Mestre, Chefe dos Setores de Cirurgia Plástica Ocular e Órbita da Disciplina de Oftalmologia da Faculdade de Medicina do ABC FMABC - Santo André (SP) - Brasil;

${ }^{4}$ Professor Titular e Chefe da Disciplina de Oftalmologia da Faculdade de Medicina do ABC - FMABC - Santo André (SP) - Brasil;

Trabalho desenvolvido na Disciplina de Oftalmologia da Faculdade de Medicina do ABC - FMABC - Santo André (SP) - Brasil.
}

Recebido para publicação em: 6/10/2009 - Aceito para publicação em 9/12/2009 


\section{INTRODUÇÃO}

$\Delta$ órbita é uma estrutura óssea piramidal que contém o globo ocular, músculos extraoculares, gordura, vasos e nervos ${ }^{(1)}$. Qualquer processo patológico que produza um aumento do volume de seu conteúdo resultará em protusão do globo ocular ${ }^{(2-4)}$.A proptose é um dos sinais mais precoces nos casos de glioma, retinoblastoma e rabdomiossarcoma ${ }^{(4)}$, e auxilia o diagnóstico de diversas doenças orbitárias como deformidades congênitas, oftalmopatia de Graves, fraturas "blow-in" ou "blow-out"(5).

Dentre os métodos para se avaliar o grau de proptose ocular, o exoftalmômetro de Hertel é o mais utilizado $^{(6,7)}$. Esse método permite medir a distância entre os rebordos orbitários laterais (base) e a exoftalmometria, simultaneamente. A exoftalmometria representa a distância entre a rima orbitária lateral e o ápice da córnea perpendicular ao plano frontal (figura 1 ).

Estabelecer uma faixa de normalidade auxilia o diagnóstico das doenças orbitárias, e evita investigações desnecessárias.

Os valores da exoftalmometria do olho humano considerados normais variam entre 10 e $21 \mathrm{~mm}^{(8)}$. No entanto, as médias da exoftalmometria no adulto normal com o exoftalmômetro de Hertel, realizadas em vários países, apresentam variações ${ }^{(9,10)}$. Evidências antropológicas mostram que a estrutura esquelética pode diferir entre as raças e também entre os sexos ${ }^{(4)}$. Outros fatores que também podem influenciar na medida da exoftalmometria são a idade, o erro refrativo e a distância entre os rebordos orbitários externos.

O objetivo deste trabalho é estabelecer os valores médios da exoftalmometria e da distância entre os rebordos orbitários externos (base), comparando sexo e raça na população adulta da Região do Grande ABC São Paulo/Brasil.

\section{Métodos}

Foram selecionados 204 pacientes da região do Grande ABC, que procuraram o Serviço de Oftalmologia da Faculdade de Medicina do ABC durante o ano de 2007, na faixa etária de 25 a 55 anos, média de 44,76 anos. Destes, 129 (63,2\%) eram do sexo feminino e 75 $(36,8 \%)$ do sexo masculino.

Os pacientes foram separados em três grupos raciais (brancos, negros e pardos), conforme a classificação utilizada pelo Instituto Brasileiro de Geografia e Estatística (IBGE). Neste, consideraram-se cinco categorias para a pessoa se classificar quanto à característica cor ou raça: branca, preta, amarela, parda (incluindose nesta categoria a pessoa que se declarou mulata, cabocla, cafuza, mameluca ou mestiça de preto com pessoa de outra cor ou raça) e indígena (caso a pessoa de-

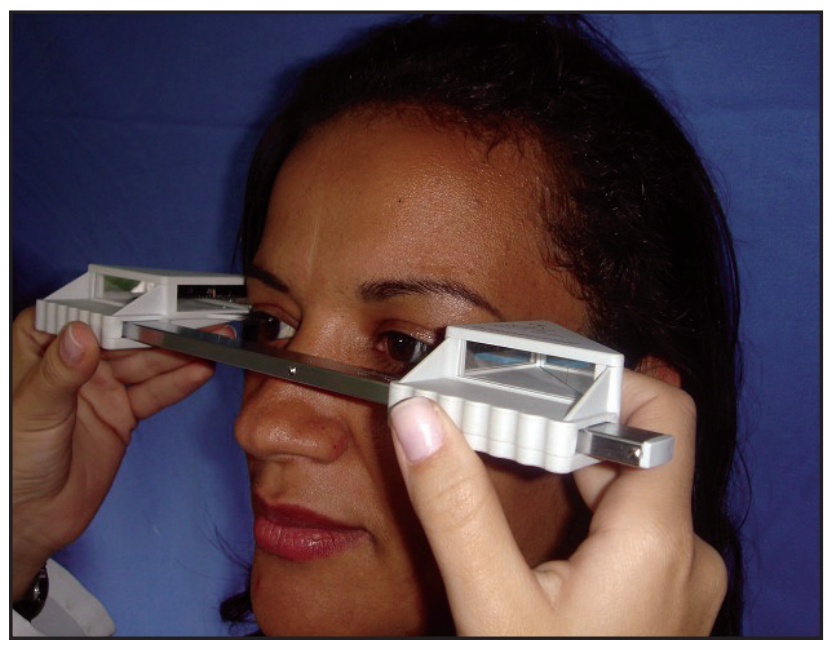

Figura 1: Técnica de medida da exoftalmometria e da base com exoftalmômetro de Hertel

clarou-se indígena ou índia) ${ }^{(11)}$. Conforme a classificação do IBGE, $90(44,1 \%$ ) pacientes se declararam brancos, 53 (26,0\%) negros e 61 (29,9\%) pardos. A distribuição dos pacientes por raça e sexo está na tabela 1.

Neste trabalho os critérios de exclusão foram: acuidade visual menor que $0,3 \log$ MAR; história prévia de cirurgia palpebral, orbitária e/ou facial; outra doença palpebral e/ou orbitária; antecedentes pessoais de tireoidopatia e doenças corneanas; pacientes com alteração no diâmetro antero-posterior do globo ocular, tais como alta hipermetropia ou alta miopia (+3,00 a -3,00D), phthisis bulbi, buphthalmo e microftalmo.

Um único pesquisador realizou a medida da exoftalmometria e da base de todos os pacientes através do exoftalmômetro de Hertel. Os pacientes foram posicionados com os olhos na mesma altura dos olhos do observador em posição primária do olhar.As plataformas do exoftalmômetro foram apoiadas nas bordas laterais da órbita, evitando-se uma pressão excessiva e mantendo-o paralelo ao plano horizontal. Observou-se a distância em milímetros entre a rima orbitária lateral e o ápice da córnea perpendicular ao plano frontal. A base foi medida observando-se diretamente a escala em milímetros do exoftalmômetro.

Cada participante foi devidamente orientado sobre o estudo e assinou o Termo de Consentimento Livre e Esclarecido de forma voluntária. O estudo foi revisado e aprovado pelo Comitê de Ética em Pesquisa da Faculdade de Medicina do ABC.

Foi realizada a análise descritiva de todas as variáveis do estudo. As variáveis qualitativas foram apresentadas em termos de seus valores absolutos e relativos e as variáveis quantitativas foram apresentadas em termos de seus valores de tendência central e de dispersão. Para se comparar os dois grupos foi utilizado o Teste $t$, pois as variáveis apresentavam distribuição normal (teste de 
Kolmogorov-Smirnov) e variâncias homogêneas (teste de Levene). Para se comparar os três grupos foi utilizado os testes de ANOVA (analise de variância) no caso paramétrico e Teste de Kruskal-Wallis no caso não paramétrico. A seguir foi utilizado o teste de comparações múltiplas de Tukey. Para se verificar a associação entre as variáveis qualitativas foi utilizado o teste de Quiquadrado. O nível de significância adotado foi de 5\%. Pacote estatístico utilizado SPSS 14.0 for Windows.

\section{Resultados}

Os valores das exoftalmometrias encontradas na população em estudo variaram entre 10 e $23 \mathrm{~mm}$, média de $15,57 \pm 2,43 \mathrm{~mm}$. No sexo feminino a média da exoftalmometria foi de $15,42 \pm 2,40 \mathrm{~mm}$ e no masculino, $15,83 \pm 2,47 \mathrm{~mm}$. Não foi encontrada uma diferença estatisticamente significativa entre esses dois grupos $(\mathrm{p}=0,25)$

$\mathrm{Na}$ raça branca a média da exoftalmometria foi de $15,57 \pm 2,46 \mathrm{~mm}$, na negra foi de $16,20 \pm 2,70 \mathrm{~mm}$ e na parda foi de $15,02 \pm 2,01 \mathrm{~mm}$. Não houve diferença estatisticamente significativa entre as raças branca e negra $(\mathrm{p}=0,110)$ e entre as raças branca e parda $(\mathrm{p}=0,180)$. Houve diferença estatisticamente significativa entre as raças negra e parda $(\mathrm{p}=0,008)$ (tabela 2$)$.

A medida da base variou entre 104 e $125 \mathrm{~mm}$ no grupo estudado, sendo a média de $113,91 \pm 4,37 \mathrm{~mm}$. Nas mulheres a média foi de $112,58 \pm 3,96 \mathrm{~mm}$ e nos homens, de 116,20 $\pm 4,10 \mathrm{~mm}$. Esta diferença foi estatisticamente significativa $(\mathrm{p}<0,001)$. Na raça branca a média da base encontrada foi de $112,18 \pm 4,02 \mathrm{~mm}$, na negra foi de 115,37 $\pm 4,71 \mathrm{~mm}$ e na parda, $115,18 \pm 3,66 \mathrm{~mm}$. Entre as raças branca e negra e entre branca e parda, a diferença da média da base foi estatisticamente significativa $(p<0,001)$. Não houve diferença estatisticamente significativa entre as raças negra e parda $(p=0,799)$.

\section{Tabela 1}

\section{Distribuição dos pacientes estudados} conforme sexo e raça

\begin{tabular}{lccc}
\hline & Feminino & Masculino & TotalN(\%) \\
\hline Branco & 67 & 23 & $90(44,1)$ \\
Negro & 30 & 23 & $53(26,0)$ \\
Pardo & 32 & 29 & $61(29,9)$ \\
Total & 129 & 75 & $204(100)$ \\
\hline
\end{tabular}

Tabela 2

Valores médios e desvio padrão da exoftalmometria e da base em milímetros, nos grupos estudados

\begin{tabular}{llcc}
\hline & & Exoftalmometria & Base \\
\hline \multirow{4}{*}{ Feminino } & Branco & $15,35 \pm 2,26$ & $111,46 \pm 3,84$ \\
& Negro & $16,03 \pm 3,01$ & $113,30 \pm 3,93$ \\
& Pardo & $14,98 \pm 2,00$ & $114,25 \pm 3,61$ \\
\multirow{2}{*}{ Masculino } & Branco & $16,22 \pm 2,86$ & $114,30 \pm 3,86$ \\
& Negro & $16,43 \pm 2,27$ & $118,08 \pm 4,31$ \\
& Pardo & $15,06 \pm 2,12$ & $116,20 \pm 3,49$ \\
\hline
\end{tabular}

Tabela 3

Comparação da exoftalmometria entre diferentes grupos populacionais; média e desvio padrão

\begin{tabular}{|c|c|c|c|c|c|}
\hline População & Idade & Sexo & $\begin{array}{c}\text { Média da } \\
\text { Exoftalmometria } \\
(\mathbf{m m})\end{array}$ & $\begin{array}{l}\text { Desvio } \\
\text { padrão }\end{array}$ & Investigador \\
\hline \multirow[t]{2}{*}{ Brasileiros do Grande ABC } & $25-55$ & M & 15,83 & 2,47 & presente estudo \\
\hline & & $\mathrm{F}$ & 15,42 & 2,40 & \\
\hline \multirow[t]{2}{*}{ Negros Americanos } & $18-91$ & M & 18,56 & 3,08 & Migliori e Gladstone ${ }^{(13)}$ \\
\hline & & $\mathrm{F}$ & 17,90 & 2,61 & \\
\hline \multirow[t]{2}{*}{ Brancos Americanos } & $18-91$ & M & 16,55 & 2,57 & Migliori e Gladstone ${ }^{(13)}$ \\
\hline & & $\mathrm{F}$ & 15,46 & 2,34 & \\
\hline \multirow[t]{2}{*}{ Brancos Europeus } & $20-80$ & M & 16,50 & 2,30 & Fledelius e Stubgaard ${ }^{(19)}$ \\
\hline & & $\mathrm{F}$ & 16,00 & 1,79 & \\
\hline \multirow[t]{2}{*}{ Nordeste da Turquia } & $3-80$ & M & 13,49 & 2,60 & Bilen, Gullulu, Akcay ${ }^{(4)}$ \\
\hline & & $\mathrm{F}$ & 13,39 & 2,60 & \\
\hline \multirow[t]{2}{*}{ Chineses de Hong Kong } & $18-60$ & M & 16,73 & 1,90 & Quant e Woo ${ }^{(26)}$ \\
\hline & & $\mathrm{F}$ & 16,64 & 1,81 & \\
\hline \multirow[t]{2}{*}{ Mexicanos } & $20-62$ & M & 15,18 & 2,10 & Gil de Montes, Pérez Resinas, \\
\hline & & $\mathrm{F}$ & 14,82 & 1,98 & Rodríguez García, González Ortiz ${ }^{(32)}$ \\
\hline \multirow[t]{2}{*}{ Chineses de Taiwan } & $18-90$ & M & 13,97 & 2,23 & Tsai, Kau, Kao e Hsu ${ }^{(15)}$ \\
\hline & & $\mathrm{F}$ & 13,86 & 2,46 & \\
\hline
\end{tabular}


Com relação à diferença entre a medida da exoftalmometria dos dois olhos, dos 204 pacientes estudados, 42 apresentaram alguma assimetria. Destes, 39 apresentaram $1 \mathrm{~mm}$ de assimetria e 3 pacientes apresentaram $2 \mathrm{~mm}$. Nenhum paciente apresentou diferença nos valores entre os dois olhos maior que $2 \mathrm{~mm}$.

\section{DiscuSSÃO}

Há mais de 40 tipos de exoftalmômetros descritos na literatura desde $1865^{(9)}$. Dentre eles, os exoftalmômetros de Lued, Mutch e Hertel avaliam a protrusão ocular através da margem orbitária lateral ${ }^{(4)}$. Atualmente, o exoftalmômetro de Hertel, descrito pela primeira vez em 1905, é o mais utilizado ${ }^{(12)}$.

O exoftalmômetro de Hertel é um aparelho familiar, portátil e acessível. Ele mede a distância do ápice da córnea à rima lateral orbitária, perpendicular ao plano frontal, através de um sistema de espelhos e uma escala milimétrica. A exoftalmometria dos dois olhos é feita simultaneamente. O aparelho mede também a distância entre as rimas orbitárias laterais. ${ }^{(13)} \mathrm{O}$ erro nas medidas ocorre principalmente pelo mal posicionamento das plataformas ${ }^{(14)}$, que pode ser minimizado estabelecendo a distância da base obtida nas consultas anteriores ${ }^{(13)}$. Um erro na medida da base resulta em diferença na exoftalmometria. A compressão excessiva contra os tecidos periorbitários pode acarretar em alterações de até $1 \mathrm{~mm}$ na medição ${ }^{(15)}$. Esse erro pode aumentar quando há um edema ao redor da margem orbitária lateral, como na oftalmopatia de Graves ${ }^{(7)}$. Estudos anteriores mostraram que os resultados da exoftalmometria realizada no mesmo grupo de pacientes tende a variar entre diferentes pesquisadores. Neste estudo, para minimizar tal efeito, todas as medidas foram realizadas por um mesmo pesquisador e evitando-se a compressão excessiva da margem orbitária lateral.

A exoftalmometria é absoluta, se confrontada com um padrão de normalidade; comparativa, quando comparada com outra medida do mesmo paciente, tomada em oportunidades diferentes; e relativa, se comparada com o valor do olho contralateral ${ }^{(16)}$.

A proptose é um sinal comum de doença orbitária, tumores envolvendo a órbita ou o crânio. A oftalmopatia de Graves é uma causa comum de exoftalmia devido ao edema e fibrose da musculatura extraocular e pela proliferação da gordura periorbitária e tecido conectivo. O enoftalmo é uma redução da protrusão do globo ocular que pode ocorrer em algumas anormalidades crâniofaciais ou após traumas orbitários ${ }^{(13)}$. O manejo médico para essas condições depende da comparação com valores previamente conhecidos da exoftalmometria de indivíduos com a mesma origem étnica, idade e sexo, para monitorar e orientar a necessidade de futuras investigações.

Os valores de normalidade da exoftalmometria encontrados na literatura para a população caucasiana variam entre 10 e $22 \mathrm{~mm}^{(17)}$, semelhante ao resultado deste estudo que mostrou uma variação entre 10 e $23 \mathrm{~mm}$.

Evidências quanto à influência do sexo em relação à exoftalmometria são ambíguas (Tabela 3). Migliori e Gladstone em 1984 e Dunsky em 1992 encontraram diferenças entre homens e mulheres nos valores da exoftalmometria. ${ }^{(13,18)}$ No entanto, Tsai et al. ${ }^{(15)}$ não encontraram uma diferença significativa entre os sexos na população chinesa estudada, sendo $13,97 \pm 2,26 \mathrm{~mm}$ a média encontrada para os homens e 13,86 $\pm 2,39$, a das mulheres. Nucci P et al. em 1989 e Fledelius e Stubgaard em 1986 também não encontraram diferenças entre os valores entre homens e mulheres ${ }^{(17,19)}$. Observou-se neste estudo para o sexo feminino uma média de 15,42 $\pm 2,40$ e para o masculino, $15,83 \pm 2,47$. A diferença entre os sexos também não foi estatisticamente significativa neste estudo $(p=0,25)$.

O limite superior da normalidade é tradicionalmente considerado $21 \mathrm{~mm}$. No entanto, esse limite varia conforme a população estudada: japoneses $(17,7 \mathrm{~mm})^{(20)}$, coreanos $(18,3 \mathrm{~mm})^{(7)}$, iranianos $(19,3 \mathrm{~mm})^{(21)}$, e chineses $(18,6 \mathrm{~mm})^{(15)}$. Observou-se neste estudo um limite superior de $20 \mathrm{~mm}$ para os brancos e os pardos, e de $23 \mathrm{~mm}$ para o grupo dos negros. Esses achados condizem com a literatura. Migliori e Gladstone ${ }^{(13)}$ encontraram 20,1 mm e $21,7 \mathrm{~mm}$ para mulheres e homens brancos, respectivamente, $23,0 \mathrm{~mm}$ para mulheres negras e $23,7 \mathrm{~mm}$ para homens negros. Pacientes com valores fora destes limites, na população estudada, devem ser investigados sobre o ponto de vista endocrinológico e radiológico.

Diversos autores encontraram uma média aumentada da protrusão ocular em negros comparado com os brancos $^{(3,2)}$ Brown e Douglas ${ }^{(3)}$ encontraram médias de $17 \mathrm{~mm}$ para os adultos negros e $14 \mathrm{~mm}$ para os brancos estudados. Migliori e Gladstone encontraram 18,5mm e 16,5mm para negros e brancos americanos, respectivamente ${ }^{(13)}$. No entanto, Cole et al. ${ }^{(23)}$ não encontraram em seu estudo uma diferença significativa $(p=0,461)$ entre brancos $(17,34 \mathrm{~mm}$ nos homens e $17,47 \mathrm{~mm}$ nas mulheres) e negros $(18,57 \mathrm{~mm}$ em homens e 19,23 em mulheres). Essa diferença também não foi estatisticamente significativa neste estudo. Nos indivíduos da raça parda, com uma média de 15,02 $\pm 2,01$, a diferença foi estatisticamente significativa quando comparada com o grupo da raça negra $(\mathrm{p}=0,008)$. Na literatura não encontramos referências sobre a média da exoftalmometria em adultos pardos.

Há um consenso geral de que a exoftalmometria aumenta do nascimento ao final da adolescência ${ }^{(19,24)}$. A partir desta idade os resultados são conflitantes. Alguns autores observaram um aumento contínuo do grau de protrusão devido ao aumento da gordura periorbitária ${ }^{(25)}$, outros observaram uma estabilização após o final da adolescência $^{(19)}$ e outros, um decréscimo após os 20 $\operatorname{anos}^{(19,26)}$. Os pacientes incluídos neste estudo encontravam-se na faixa etária de 25 a 55 anos. 
Com relação ao erro refrativo, graus leves a moderados $(+6,00 \mathrm{D}$ a $-6,00 \mathrm{D})$ não apresentaram efeito significativo na protrusão ${ }^{(13,19,27)}$. No entanto, diversos autores excluíram pacientes alto míopes de seus estudos para que o aumento no diâmetro axial, relacionado com miopias axiais altas, não influenciassem nos resultados. Quant e $\mathrm{Woo}^{(26)}$ observaram que os pacientes com alta miopia (maior que $5,00 \mathrm{D}$ ) apresentavam médias de exoftalmometria mais elevadas. Além disso, consideraram essa diferença especialmente importante em casos de anisometropia, podendo gerar valores assimétricos para a exoftalmometria de cada olho. Os pacientes selecionados para este estudo tinham seu erro refrativo entre $+3,00 \mathrm{D}$ e $-3,00 \mathrm{D}$.

Algumas dimensões facias se correlacionam com o grau de protrusão, entre elas a distância entre os rebordos orbitários externos, sendo que uma diminuição da mesma acarreta em uma diminuição no grau de protrusão ocular $^{(19)}$. O hipertelorismo é o termo usado para descrever um aumento anormal da distância entre as órbitas pela parte óssea e o telecanto por partes moles. Algumas doenças sistêmicas estão associadas a anormalidades crâniofaciais como a síndrome de Alpert e Crouzon. O hipotelorismo é uma distância menor que a normal entre as órbitas. Fatores como o aumento na distância entre as sobrancelhas, prega epicântica alargada ou ponte nasal chata, podem dar uma falsa impressão clínica de hipertelorismo ${ }^{(26)}$. Apesar de as anormalidades faciais serem normalmente congênitas, o manejo no adulto, como em casos de correção cirúrgica, necessita do conhecimento de valores de normalidade nesta faixa etária.

Neste estudo, a média da base foi de 113,91 $\pm 4,37 \mathrm{~mm}$. Em brancos americanos a medida da base encontrada foi de $98,39 \pm 3,86 \mathrm{~mm}$ no sexo masculino e de $95,41 \pm 3,83 \mathrm{~mm}$ no sexo feminino e em negros americanos de $98,86 \pm 3,57 \mathrm{~mm}$ em homens e de $95,70 \pm 3,44 \mathrm{~mm}$ em mulheres ${ }^{(18)}$. Neste estudo, a média na raça branca foi de $111,46 \pm 3,84 \mathrm{~mm}$ no sexo feminino e de $114,30 \pm 3,86 \mathrm{~mm}$ no masculino; na raça negra, a média foi de $113,30 \pm 3,93 \mathrm{~mm}$ no sexo feminino e de $118,08 \pm 4,31 \mathrm{~mm}$ no masculino. Estudos antropológicos realizados no final do século 19 mostraram que negros têm uma órbita retangular, orientais uma órbita mais arredondada e os brancos algo intermediário ${ }^{(28)}$. Bogren et al. ${ }^{(29)}$ observaram, através da exoftalmometria radiográfica, que a posição ocular está diretamente relacionada com o tamanho do crânio e o grau de protrusão ocular, e que os indivíduos da raça negra têm um grau de protrusão maior para um mesmo tamanho de crânio comparado com os de raça branca. A média da protrusão ocular mais elevada e a maior distância interorbital medida na raça negra é resultado de órbitas mais rasas e possivelmente um aumento no ângulo entre os eixos longitudinais da órbita, comparado com a raça branca. A diferença antropológica deve explicar a grande variação da exoftalmometria e da base entre os diferentes grupos populacionais. Entre as raças estudadas, a branca apresentou valores estatisticamente menores da base, sendo a média nesta raça de $112,18 \mathrm{~mm}$, enquanto que na raça negra e parda ela foi de $115,37 \mathrm{~mm}$ e $115,18 \mathrm{~mm}$, respectivamente.

A maioria dos nossos pacientes $(79,42 \%)$ não apresentou assimetria na medida absoluta da protrusão ocular entre os olhos direito e esquerdo. Esse achado é compatível com o encontrado em outros estudos: $69 \%^{(19)}$, $70 \%{ }^{(26)}, 72 \%{ }^{(26)} 76,4 \%{ }^{(30)}$. Migliori e Gladstone ${ }^{(13)}$ encontraram uma exoftalmometria relativa máxima de $2,0 \mathrm{~mm}$ tanto para americanos da raça branca como da negra estudados . Alguns estudos mostram que negros apresentam uma exoftalmometria relativa maior, de $3,5 \mathrm{~mm}^{(29)}$ e $3,0 \mathrm{~mm}^{(22)}$. Nenhum paciente do estudo apresentou uma assimetria maior de $2,0 \mathrm{~mm}$ e não houve uma relação entre o valor da assimetria e a raça.

A diferença na protrusão absoluta entre os dois olhos pode ser importante no diagnóstico de proptose unilateral. Soroudi et al. ${ }^{(31)}$ observaram que $9 \%$ dos pacientes com orbitopatia relacionada à tireóide apresentaram uma proptose assimétrica $\geq 2 \mathrm{~mm}$. Uma assimetria maior que $2 \mathrm{~mm}$ em indivíduos da população estudada, deve ser investigada.

Este estudo assume importância clínica considerando-se a falta de uma faixa de normalidade da exoftalmometria na população brasileira. No entanto, os valores encontrados referem-se somente a uma amostra da população brasileira habitante da região do Grande ABC.Assim, considerando-se ainda a heterogeneidade da população brasileira, os valores encontrados não se aplicam a toda população. Estudos mais abrangentes e que analisem as diversas regiões do país se fazem necessários.

\section{Conclusão}

1- Foram observados os seguintes valores médios da exoftalmometria na população adulta da região do Grande ABC, São Paulo/Brasil conforme raça e sexo: Raça branca, sexo feminino: $15,35 \pm 2,26 \mathrm{~mm}$ Raça branca, sexo masculino: $16,22 \pm 2,86 \mathrm{~mm}$ Raça parda, sexo feminino: $14,98 \pm 2,00 \mathrm{~mm}$ Raça parda, sexo masculino: $15,06 \pm 2,12 \mathrm{~mm}$ Raça negra, sexo feminino: $16,03 \pm 3,01 \mathrm{~mm}$ Raça negra, sexo masculino: $16,43 \pm 2,27 \mathrm{~mm}$

2- Foram observados os seguintes valores médios da distância entre os rebordos orbitários externos (base) na população adulta da região do Grande $\mathrm{ABC}$, São Paulo/Brasil conforme a raça e sexo:

Raça branca, sexo feminino: $111,46 \pm 3,84 \mathrm{~mm}$

Raça branca, sexo masculino: $114,30 \pm 3,86 \mathrm{~mm}$

Raça parda, sexo feminino: $114,25 \pm 3,61 \mathrm{~mm}$

Raça parda, sexo masculino: $116,20 \pm 3,49 \mathrm{~mm}$

Raça negra, sexo feminino: $113,30 \pm 3,93 \mathrm{~mm}$

Raça negra, sexo masculino: $118,08 \pm 4,31 \mathrm{~mm}$ 


\begin{abstract}
Purpose: To determine the normal exophthalmometry of both eyes and of the distance between lateral orbital rims (the base measurement) in young adults from ABC Region, São Paulo/ Brazil. Methods: Exophthalmometry, by Hertel's exophthalmometer, was made in 129 female and 75 male subjects, age ranging from 25 to 55 years. The subjects were divided in three groups of races (white, black and mulatto). Results: The values of exophthalmometry ranged from 10 to $23 \mathrm{~mm}$, mean $15,57 \pm 2,43 \mathrm{~mm}$. In female the mean was 15,42 $\pm 2,40 \mathrm{~mm}$ and in male, $15,83 \pm 2,47 \mathrm{~mm}$. There is no statistically significant difference between female or male $(p=0,25)$. In whites subjects the mean was $15,57 \pm 2,46 \mathrm{~mm}$, in black was $16,20 \pm 2,70 \mathrm{~mm}$ and in mulatto was 15,02 $\pm 2,01 \mathrm{~mm}$. There was a statistically significant difference between blaks and mulattos $(p=0,008)$. The base measurement ranged from 104 to $125 \mathrm{~mm}$ (mean $113,91 \pm 4,37 \mathrm{~mm}$ ). There was a statistically significant difference between males and females $(p<0,001)$. In whites the base was 112,18 $\pm 4,02 \mathrm{~mm}$, in blacks 115,37 $\pm 4,71 \mathrm{~mm}$ and in mulattos, $115,18 \pm 3,66 \mathrm{~mm}$. There was a statistically significant difference for the base measurement between white subjects and black ones, and between white subjects and mulattos $(p<0,001)$. No individual had more than $2 \mathrm{~mm}$ of assimetry between eyes. Conclusion: The normal exophthalmometric value in the studied population was 10 to $23 \mathrm{~mm}$ and the base measurement ranged from 104 to $125 \mathrm{~mm}$.

Keywords: Exophthalmos/diagnosis; Diagnostic techniques, ophthalmologicalinstrumentation; Reference values

\section{ReferenCias}

1. Testut L, Latarget A. Tratado de anatomia humana. Barcelona: Salvat; 1967.

2. Char DH. Eye signs and diagnosis of thyroid ophthalmopathy. In: Char DH. Thyroid eye disease. New York: Churchill Livingstone; 1990. p. 35.

3. Brown RD, Douglas J. Letter: Exophthalmometry in blacks. Ann Intern Med. 1975;83(6):835-6.

4. Bilen H, Gullulu G, Akcay G. Exophthalmometric values in a normal Turkish population living in the northeastern part of Turkey. Thyroid. 2007;17(6):525-8.

5. Barreto RL, Mathog RH. Orbital measurement in black and white populations. Laryngoscope. 1999;109(7 Pt 1):1051-4.

6. Chang AA, Bank A, Francis IC, Kappagoda MB. Clinical exophthalmometry: a comparative study of the Luedde and Hertel exophthalmometers. Aust N Z J Ophthalmol. 1995;23(4):315-8.

7. Kim IT, Choi JB. Normal range of exophthalmos values on orbit computerized tomography in Koreans. Ophthalmologica. 2001;215(3):156-62.

8. Wright JE. Proptosis. Ann R Coll Surg Engl. 1970;47(6):323-34.

9. Sleep TJ, Manners RM. Interinstrument variability in Herteltype exophthalmometers. Ophthal Plast Reconstr Surg. 2002;18(4):254-7.

10. Rodrigues Alves CA. Contribuição ao estudo da exoftalmometria: resultados de 704 medições com exoftalmometro de Hertel [tese]. São Paulo: Faculdade de Medicina da Universidade de São Paulo; 1981.

11. Instituto Brasileiro de Geografia e Estatística - IBGE. Pesquisa Nacional por Amostra de Domicílios - PNAD 1999. Conceituação das características investigadas, cor ou raça.[Internet]. [citado 2009 Dez 12]. Disponível em: http:// www.ibge.gov.br/home/estatistica/populaca o/ trabalhoerendimento/ pnad99/metodologia99.shtm
12. Mourits MP, Lombardo SH, van der Sluijs FA, Fenton S. Reliability of exophthalmos measurement and the exophthalmometry value distribution in a healthy Dutch population and in Graves' patients. An exploratory study. Orbit. 2004;23(3):161-8. Comment in: Orbit. 2004;23(3):147-51.

13. Migliori ME, Gladstone GJ. Determination of the normal range of exophthalmometric values for black and white adults. Am J Ophthalmol. 1984;98(4):438-42.

14. Davanger M. Principles and sources of error in exophthalmometry. A new exophthalmometer. Acta Ophthalmol (Copenh). 1970;48(4):625-33.

15. Tsai CC, Kau HC, Kao SC, Hsu WM. Exophthalmos of patients with Graves' disease in Chinese of Taiwan. Eye. 2006;20(5):569-73.

16. Gonçalves JOR. Exoftalmometria. Rev Bras Oftalmol. 1979;38:331-42.

17. Nucci P, Brancato R, Bandello F, Alfarano R, Bianchi S. Normal exophthalmometric values in children. Am J Ophthalmol. 1989;108(5):582-4.

18. Dunsky IL. Normative data for hertel exophthalmometry in a normal adult black population. Optom Vis Sci. 1992;69(7):562-4.

19. Fledelius HC, Stubgaard M. Changes in eye position during growth and adult life as based on exophthalmometry, interpupillary distance, and orbital distance measurements. Acta Ophthalmol (Copenh). 1986;64(5):481-6.

20. Amino N, Yuasa T, Yabu Y, Miyai K, Kumahara Y. Exophthalmos in autoimmune thyreoid disease. J Clin Endocrinol Metab. 1980;51(6):1232-4.

21. Kashkouli MB, Beigi B, Noorani MM, Nojoomi M. Hertel exophthalmometry: reliability and interobserver variation. Orbit. 2003;22(4):239-45.

22. de Juan E Jr, Hurley DP, Sapira JD. Racial differences in normal values of proptosis. Arch Intern Med. 1980;140(9):1230-1.

23. Cole HP 3rd, Couvillion JT, Fink AJ, Haik BG, Kastl PR. Exophthalmometry: a comparative study of the Naugle and Hertel instruments. Ophthal Plast Reconstr Surg. 1997;13(3):189-94. Comment in: Ophthal Plast Reconstr Surg. 1999;15(4):299-301.

24. Kaye SB, Green JR, Luck J, Lowe KJ. Dependence of ocular protrusion, assimetry of protrusion and lateral interobital width on age. Acta Ophthalmol (Copenh). 1992;70(6):762-5.

25. Knudtzon K. On exophthalmometry; the result of 724 measurements with Hertel's exophthalmometer on normal adult individuals. Acta Psychiatr Neurol. 1949;24(3-4):523-37.

26. Quant JR, Woo GC. Normal values of eye position in the Chinese population of Hong Kong. Optom Vis Sci. 1992;69(2):152-8.

27. Quant JR, Woo GC. Eye position and head size in the Chinese population: a comparison of the Chinese from Hong Kong with the Chinese from Guangdong Province. Optom Vis Sci. 1992;69(10):793-6.

28. Duke-Elder S, MacFaul PA. The ocular adnexa. Lacrimal, orbital and para-orbital diseases. In: Duke-Elder WS, editors. System of ophthalmology. St Luis: CV Mosby; 1974. 13, pt2, p. 780-5.

29. Bogren HG, Schermer MJ, Franti C, Elfström G, Tengroth B. Radiographic exophthalmometry. Trans Sect Ophthalmol Am Acad Ophthalmol Otolaryngol. 1976;81(2):298-304.

30. Osuobeni EP, al-Harbi AA. Normal values of ocular protrusion in Saudi Arabian male children. Opt Vis Sci.1995,72(8):557-64.

31. Soroudi AE, Goldberg RA, McCann JD. Prevalence of assymmetric exophthalmos in Graves orbitopathy. Ophthal Plast Reconstr Surg. 2004;20(3):224-5.

32. Bolaños Gil de Montes F, Pérez Resinas FM, Rodríguez García M, González Ortiz M. Exophthalmometry in Mexican adults. Rev Invest Clin. 1999;51(6):341-3.

\section{Endereço para correspondência: \\ Nadia Ajub Moysés}

Rua dos Ingleses, $\mathrm{n}^{\circ} 222$ - Apto.111

CEP 01329-000 - São Paulo (SP), Brasil

E-mail: nadiaajub@gmail.com 